EESTI NSV TEADUSTE AKADEEMIA TOIMETISED.

FOOSIKA * MATEMAATIKA

ИЗВЕСТИЯ АКАДЕМИИ НАУК ЭСТОНСКОН ССР. ФИЗИКА * МАТЕМАТИКА

PROCEEDINGS OF THE ACADEMY OF SCIENCES OF THE ESTONIAN SSR.

PHYSICS * MATHEMATICS

1986. 35,4

удК 534.2

H. ВЕКСЛЕР

\title{
СТАЦИОНАРНОЕ РАССЕЯНИЕ АКУСТИЧЕСКОЙ ВОЛНЫ ТОЛСТОСТЕННЫМ КРУГОВЫМ УПРУГИМ ЦИЛИНДРОМ
}

\author{
(Представил Н. Алумяэ)
}

Исследуется процесс рассеяния плоской акустической волны давления толстостенным круговым цилиндром. Направление распространения волны перпенднкулярно продольной оси цилиндра, так что задача является двумерной. В точке наблюдения в дальнем поле в направлении на источник выполняется анализ частотной зависимости (ЧЗ) акустического давления, рассеянного цилиндром в жидкость. Определен состав волн, формирующих ЧЗ, и выявлена зависимость их вклада в ЧЗ от волнового радиуса. В широком диапазоне волнового радиуса идентифицированы резонансы волн различных типов на кривой ЧЗ. Приведены дисперсионные кривые для волны $A_{1}$. Уетановлено, что к моменту возбуждения волны $S_{1}$ амплитуда волны $A_{1}$ достигает максимального значения. В типичной ситуащии металлического цилиндра в воде это приводит к глубокому и широкому «провалу» крнвой ЧЗ в этой области.

Разработка аналитической процедуры, получившей название резонансной теории рассеяния (РТP) $\left[{ }^{1-7}\right]$, и экспериментального метода выделения и идентификации резонансов (МВИР) $\left.{ }^{[8-15}\right]$ во многом способствовали возрождению интереса к задачам рассеяния акустических волн толстостенными упругими телами цилиндрической и сферической формы. С их помощью удается провести интерпретацию волнового поля в жидкости, вызванного колебаниями упругого тела. К настоящему времени имеются рассчитанные и измеренные акустические спектрограммы толстостенных оболочек, построены траектории полюсов Редже для различных типов периферических волн, рассчитаны и измерены дисперсионные кривые.

Зависимость амплитуд периферических волн от волнового радиуса и результат взаимодействия волн различных типов (с учетом их фаз) изучены не столь хорошо. Цель данной заметки состоит в том, чтобы привлечь внимание исследователей к тому диапазону ЧЗ акустического давления, где начинает возбуждаться первая симметричная волна типа Лэмба $S_{1}$. Здесь амплитуда первой антисимметричной волны типа Лэмба $A_{1}$ достигает наибольшего значения, а на кривой ЧЗ появляется глубокий и широкий «провал». Он был замечен нами давно ([ $\left.{ }^{16}\right]$, фиг. 4.1), но надлежащей интерпретации не получил.

Толстостенная пустая упругая круговая цилиндрическая оболочка бесконечной длины погружена в безграничную идеальную сжимаемую жидкость. На нее падает и затем рассеивается плоская гармоническая волна давления, приходящая с направления $\theta=0$.

Давление в падающей волне $p_{i}$ имеет вид

$$
p_{i}=p_{*} \exp (i x) \exp (i x r \cos \theta)
$$

где

$$
x=k a, \quad k=\omega / c, \quad r=R / a .
$$

3 десь $p_{*}-$ постоянная, имеющая размерность давления, $x-$ волновой радиус, $a$ - наружный радиус оболочки, $\omega=$ частота, $c-$ скорость 
звука в жидкости, $r$ - безразмерная радиальная координата. Множитель $\exp (-i \omega t)$ в правой части (1) и в дальнейшем подразумевается. Используя разложение $\exp (i x r \cos \theta)$ в ряд, запишем (1) в форме

$$
\begin{gathered}
p_{i}=p_{*} \exp (i x) \sum_{n=0}^{\infty} \varepsilon_{n}(-i)^{n} J_{n}(x r) \cos n \theta \\
\left(\varepsilon_{0}=1, \varepsilon_{n}=2 \text { при } n \geqslant 1\right) .
\end{gathered}
$$

Рассеянное оболочкой акустическое давление $p_{s}$ можно представить в виде

$$
p_{s}=p_{*} \exp (i x) \sum_{n=0}^{\infty} A_{n} H_{n}^{(1)}(x r) \cos n \theta,
$$

где коэффициенты $A_{n}$ находятся из системы алгебраических уравнений, следующей из условий контакта $\left[{ }^{17}\right]$.

В дальнем поле, в направлении на источник, удобно ввести

$$
p(x)=p_{s} \frac{\sqrt{r(2-1 / r)}}{p_{*} \exp [i x(r-1)]} \quad(r \gg 1, \theta=0) .
$$

Будем называть $|p(x)|$ частотной зависимостью (ЧЗ) акустического давления.

На рис. 1 представлена ЧЗ, рассчитанная с шагом по $x$ равным $10 / 256$, для случая стальной оболочки в воде, при следующих значениях параметров

$$
r=10^{4}, \quad \theta=0, \quad h=(a-b) / a=1 / 8,
$$

сталь: $Q_{1}=7,7 \cdot 10^{3} \mathrm{Kr} / \mathrm{m}^{3}, c_{l}=5960 \mathrm{M} / \mathrm{c}, c_{t}=3240 \mathrm{~m} / \mathrm{c}$, вода: $\varrho=1 \cdot 10^{3} \mathrm{Kr} / \mathrm{m}^{3}, c=1493 \mathrm{~m} / \mathrm{c}$.

В (6) использованы следующие обозначения: $\varrho, \varrho_{1}-$ плотности жидкости и материала оболочки, $c_{l}, c_{t}$ - скорости распространения продольных и поперечных волн в линейной теории упругости, $b, h-$ внутренний радиус и относительная толщина оболочки.

Из рис. 1 видно, что изрезанность кривой ЧЗ увеличивается с ростом $x$. Как известно, ЧЗ формируется за счет вкладов, вносимых зеркально отраженной волной, волнами, переотраженными в цилиндрическом слое, дифрагированными волнами и переизлученными волнами. Вклад дифрагированных волн невелик и заметен только при $x \leqslant 10$. Переизлучение вызывается нормальными (типа Лэмба) волнами, обегающими оболочку по углу $\theta$. Каждая из переизлученных волн проявляется на кривой ЧЗ в виде экстремумов, следующих по $x$ с некоторым шагом, величина которого, вообще говоря, зависит от $x$.

Для интерпретации ЧЗ удобно испольљовать решение предельной задачи - о нормальных волнах в плоском упругом слое, свободном от напряжений по лицевым поверхностям. Рассмотрим эквивалентный (по толщине и материалу) плоский слой. В нем могут распространяться симметричные $(S)$ и антисимметричные $(A)$ нормальные (типа Лэмба) волны. Полное число симметричных $Q_{S}$ и антисимметричных $Q_{A}$ волн, возможных в плоском слое заданной толщины $(2 d=a-b)$ на частоте $\omega$, равно $\left[{ }^{18}\right]$

$$
\begin{aligned}
& Q_{\mathrm{S}}=1+\left[\frac{\omega d}{\pi c_{t}}\right]+\left[\frac{1}{2}+\frac{\omega d}{\pi c_{l}}\right], \\
& Q_{A}=1+\left[\frac{\omega d}{\pi c_{l}}\right]+\left[\frac{1}{2}+\frac{\omega d}{\pi c_{t}}\right] .
\end{aligned}
$$


номер резонанса волны, а

\begin{tabular}{c|c|c|c}
\hline Тип волны & $k_{t} d$ & $x=k a$ & Значение \\
\hline$A_{1}$ & $\frac{\pi}{2}$ & $\frac{\pi c_{t}}{h c}$ & 54,54 \\
$S_{1}$ & $\frac{\pi c_{l}}{2 c_{t}}$ & $\frac{\pi c_{l}}{h c}$ & 100,33 \\
$S_{2}$ & $\pi$ & $\frac{2 \pi c_{t}}{h c}$ & 109,08 \\
$A_{2}$ & $\frac{3 \pi}{2}$ & $\frac{3 \pi c_{t}}{h c}$ & 163,62
\end{tabular}

вторая $(l)$ - тип периферической волны. Пусть индекс $l=1$ задает волну $A_{0}, l=$ $=2$ - волну $S_{0}, l=3$ волну $A_{1}$. Здесь для нормальных волн в круговом цилиндрическом слое, контактирующим с жидкостью, использованы те же обозначения, что и в предельном плоском случае. В области $5 \leqslant x \leqslant 55$ хорошо видны резонансы волн $S_{0}$ и $A_{0}$. При $x \geqslant 55$ появляются резонансы

волны $A_{1}$ (изрезанность кривой ЧЗ резко увеличивается), вклад волны $A_{1}$ в ЧЗ растет с ростом $x$ и к $x \leqslant 100$ он становится преобладающим. Вклад же волн $S_{0}$ и $A_{0}$ в области $55 \leqslant x \leqslant 100$ монотонно падает с ростом $x$. При $x \geqslant 100$ в ЧЗ начинает вносить вклад волна $S_{1}$, а при $x \geqslant 109$ и волна $S_{2}$. При $x \geqslant 163$ становится заметным вклад волны $A_{2}$ (и здесь изрезанность кривой ЧЗ очень велика).

Для волн $S_{0}, A_{0}, A_{1}$ последовательные резонансы хорошо просматриваются на кривой суммарной ЧЗ уже с индекса $n=4$ и поэтому легко узнать индекс резонанса. Напротив, указать номера последовательных резонансов волн $S_{1}, S_{2}, A_{2}$ непосредственно из суммарной ЧЗ, представленной на рис. 1, затруднительно.

В принципе, понятно как найти индекс явного резонанса по ЧЗ. Для этой цели можно применить аналитический аппарат резонансной теории рассеяния $\left[{ }^{7}\right]$, либо рассчитать полярную диаграмму направленности на частоте, отвечающей резонансу, при временах, отвечающих уже закончившейся достаточно длительной посылке (в таком случае на полярной диаграмме направленности будет $2 n$ максимумов, где $n-$ индекс резонанса; см. фиг. 13 в $\left.\left[{ }^{13}\right]\right)$; либо рассчитать временную зависимость акустического давления при достаточно длительной, но конечной посылке с частотой, отвечающей резонансной (при этом окажется, что временная зависимость будет иметь вид «лестницы», причем число периодов $n$ длительностью $2 \pi / x$ в каждой из «ступенек» как раз и дает индекс резонанса; см. фиг. 3.16 в $\left.\left[{ }^{16}\right]\right)$.

При первоначальном рассмотрении из анализа ЧЗ не сразу ясно, возбудилась ли при $x \geqslant 55$ новая волна $\left(A_{1}\right)$ или же перестроилась волна $A_{0}$, поскольку групповые скорости этих волн довольно близки (групповая скорость волны приблизительно равна шагу последовательных однотипных экстремумов на кривой ЧЗ). С целью явного выделения на ЧЗ волны $A_{1}$ можно просчитать модельную задачу для ситуации, когда вклад волны $A_{0}$ в суммарную ЧЗ будет пренебрежимо мал. Для этого, сохранив все параметры оболочки, нужно задать довольно высокой скорость звука в жидкости. Приняв параметры жидкости

$$
\mathrm{Q}^{*}=\mathrm{\varrho}, \quad c^{*}=2,5 c,
$$

мы провели расчет ЧЗ. Результат вычисления представлен на рис. 2. Для удобства сопоставления результатов на рис. 2 по оси абцисс отложен волновой радиус $x$, а не естественный для данного случая волновой радиус

$$
x^{*}=k^{*} a, \quad k^{*}=\omega / c^{*} .
$$

До $x \leqslant 55$ на кривой ЧЗ рис. 2 видны резонансы волны $S_{0}$, а после $x \geqslant 55$ - преимущественно резонансы волны $A_{1}$. Резонансы волны $A_{0}$ на этой 
ส

¿ ल

$1|1| 1|1| 1|1|$

2
N
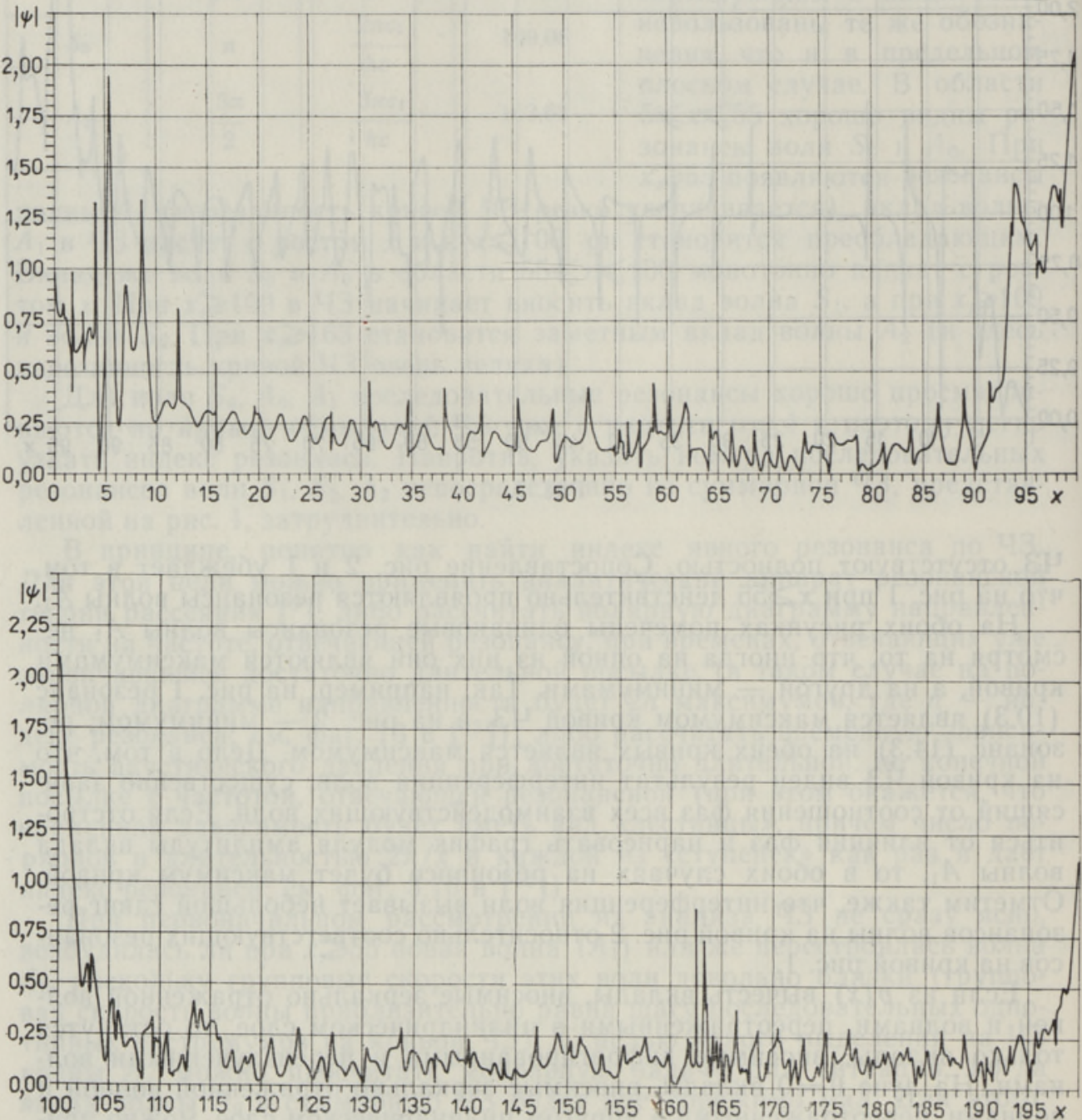

Рнс. 3.

и вычислим ее модуль. Для рассматриваемого примера на рис. 3 представлен результат вычисления $|\psi(x)|$. На кривой помечены резонансы волн $S_{0}, A_{0}, A_{1}$. Теперь в отличие от предыдущих фигур резонансы каждой из волн являются максимумами на кривой $|\psi(x)|$.

C информативной точки зрения одним из наиболее интересных диапазонов ЧЗ является окрестность точки $x=x_{*}$, в которой начинает возбуждаться волна $S_{1}:\left(k_{t} d\right)_{*}=\frac{\pi c_{l}}{2 c_{t}}$. В рассматриваемом примере $x_{*}=100,33$. Қак ясно видно из рис, 3 , амплитуды волн $S_{0}$ и $A_{0}$ падают 


\begin{tabular}{|c|c|c|c|c|c|c|c|}
\hline \multicolumn{4}{|c|}{$\begin{array}{c}h=1 / 7 \\
x_{0}=74,72 ; x_{*}=87,79\end{array}$} & \multicolumn{4}{|c|}{$\begin{array}{c}h=1 / 8 \\
x_{0}=54,54 ; x_{*}=100,33\end{array}$} \\
\hline$n$ & $x$ & $|p(x)|$ & $v_{3}{ }^{p h}$ & $n$ & $x$ & $|p(x)|$ & $v_{3}^{p h}$ \\
\hline $\begin{array}{r}4 \\
5 \\
6 \\
7 \\
8 \\
9 \\
10 \\
11 \\
12 \\
13 \\
14 \\
15 \\
16 \\
17 \\
18 \\
19 \\
20\end{array}$ & $\begin{array}{l}56,40 \\
58,59 \\
60,98 \\
63,44 \\
66,02 \\
68,48 \\
71,13 \\
73,83 \\
76,45 \\
78,95 \\
81,33 \\
83,63 \\
85,82 \\
88,13 \\
90,55 \\
93,05 \\
95,51\end{array}$ & $\begin{array}{l}0,813 \\
0,800 \\
0,855 \\
0,881 \\
0,861 \\
1,237 \\
1,206 \\
1,293 \\
1,329 \\
1,306 \\
1,658 \\
0,037 \\
0,689 \\
1,204 \\
1,309 \\
1,277 \\
0,961\end{array}$ & $\begin{array}{r}14,10 \\
11,72 \\
10,16 \\
9,06 \\
8,25 \\
7,61 \\
7,11 \\
6,71 \\
6,37 \\
6,07 \\
5,81 \\
5,58 \\
5,36 \\
5,18 \\
5,03 \\
4,90 \\
4,78\end{array}$ & $\begin{array}{r}5 \\
6 \\
7 \\
8 \\
9 \\
10 \\
11 \\
12 \\
13 \\
14 \\
15 \\
16 \\
17 \\
18 \\
19 \\
20 \\
21\end{array}$ & $\begin{array}{r}64,18 \\
66,33 \\
68,63 \\
71,02 \\
73,52 \\
76,05 \\
78,52 \\
81,17 \\
83,79 \\
86,45 \\
89,02 \\
91,48 \\
93,55 \\
96,17 \\
98,52 \\
100,90 \\
103,32\end{array}$ & $\begin{array}{l}0,780 \\
0,770 \\
0,787 \\
0,820 \\
0,826 \\
0,805 \\
1,196 \\
1,155 \\
1,227 \\
1,329 \\
1,400 \\
1,400 \\
1,538 \\
0,317 \\
0,802 \\
1,307 \\
1,357\end{array}$ & $\begin{array}{r}12,84 \\
11,06 \\
9,80 \\
8,88 \\
8,17 \\
7,61 \\
7,14 \\
6,76 \\
6,45 \\
6,18 \\
5,93 \\
5,72 \\
5,50 \\
5,34 \\
5,19 \\
5,05 \\
4,92\end{array}$ \\
\hline
\end{tabular}

\begin{tabular}{|c|c|c|c|c|c|c|c|}
\hline \multicolumn{4}{|c|}{$\begin{array}{c}h=1 / 9 \\
x_{0}=61,36 ; x_{\star}=112,87\end{array}$} & \multicolumn{4}{|c|}{$\begin{array}{c}h=1 / 10 \\
x_{0}=68,18 ; x_{*}=125,41\end{array}$} \\
\hline$n$ & $x$ & $|p(x)|$ & $v_{3}{ }^{p h}$ & $n$ & $x$ & $p(x)$ & $\left|v_{3}^{p h}\right|$ \\
\hline 7 & 74,10 & 0,610 & 10,59 & 16 & 101,21 & 1,145 & 6,33 \\
\hline 8 & 76,37 & 0,692 & 9,55 & 17 & 103,87 & 1,169 & 6,11 \\
\hline 9 & 78,71 & 0,753 & 8,75 & 18 & 106,48 & 1,189 & 5,92 \\
\hline 10 & 81,17 & 0,762 & 8,12 & 19 & 109,10 & 1,207 & 5,74 \\
\hline 11 & 83,63 & 0,707 & 7,60 & 20 & 111,68 & 1,240 & 5,58 \\
\hline 12 & 86,17 & 0,856 & 7,18 & 21 & 114,22 & 1,355 & 5,44 \\
\hline 13 & 88,55 & 1,137 & 6,81 & 22 & 116,95 & 1,874 & 5,32 \\
\hline 14 & 91,17 & 1,156 & 6,51 & 23 & 119,10 & 0,354 & 5,18 \\
\hline 15 & 93,83 & 1,187 & 6.26 & 24 & 121,80 & 0,218 & 5,08 \\
\hline 16 & 96,45 & 1,248 & 6,03 & 25 & - & - & - \\
\hline 17 & 99,06 & 1,293 & 5,83 & 26 & - & $\overline{0}$ & $\overline{777}$ \\
\hline 18 & 101,60 & 1,373 & 5,64 & 27 & 128,71 & 0,970 & 4,77 \\
\hline 19 & - & $\overline{017}$ & - & 28 & 131,21 & 0,884 & 4,69 \\
\hline 20 & 106,44 & 0,617 & 5,32 & 29 & 133,79 & 0,795 & 4,61 \\
\hline 21 & 108,83 & 0,389 & 5,18 & 30 & 136,60 & 0,689 & 4,55 \\
\hline 22 & 111,21 & 0,874 & 5,06 & 31 & 139,22 & 0,783 & 4,49 \\
\hline 23 & 113,63 & 1,490 & 4,94 & 32 & 141,91 & 0,777 & 4,43 \\
\hline
\end{tabular}

с ростом $x$ и к $x=x$ * их вклад в ЧЗ становится небольшим. Начиная с $\left(k_{t} d\right)_{0}=\frac{\pi}{2}\left(x_{0}=\frac{\pi c_{t}}{h c}\right)$ возбуждается волна $A_{1}$ и к $x=x$ * ее амплитуда достигает наибольшего значения; после $x=x_{*}$ она быстро падает. На кривой ЧЗ это обстоятельство проявляется в виде глубокого и широкого «провала». Амплитуда ЧЗ здесь падает до уровня $|p(x)| \sim 0,1$, а ширина провала занимает полосу, в которую попадают три резонанса волны $A_{1}$. Сам провал на ЧЗ объясняется тем, что в окрестности $x=x$ * волна $A_{1}$ почти противофазна сумме зеркально отраженной волны и волн, переотраженных в слое. Отметим, что в модельной задаче при скорости звука в жидкости $c^{*}=2,5$ с волна $A_{1}$ и сумма зеркально отраженной волны и волн, переотраженных в слое, почти синфазны. Именно поэтому на рис. 2 в окрестности $x=x_{*}$ наблюдаются столь большие по амплитуде резонансы. 
Характерно, что наибольшая глубина провала имеет место не точно при $x=x_{*}$, а в его окрестности, поскольку резонансы волны $A_{1}$ следуют по $x$ с некоторым шагом, и значение $x=x$ * может не совпадать с резонансом. На кривой $|\psi(x)|$ наибольшая амплитуда также имеет место вблизи $x=x_{*}$.

Располагая информацией о положении по $x$ описанного выше провала на кривой ЧЗ, легко оценить относительную толщину оболочки

$$
h=\frac{\pi c_{l}}{x_{*} c} \text {. }
$$

Погрешность вычисления $h$ по (13) не превышает пяти процентов. Подобный провал ЧЗ имеет место и при $x \sim 2 x_{*}$.

В табл. 2 приведены координаты и величины $|p(x)|$ для последовательных резонансов волны $A_{1}$ в окрестности $x=x_{*}$ для стальных оболочек в воде при $h=1 / 7,1 / 8,1 / 9,1 / 10$. волны

Как известно, относительная фазовая скорость $l$-й периферической

$$
v_{l}^{p h}(x)=\frac{c_{l}^{p h}(x)}{c}
$$

по координате $x_{n, l}$ ее резонанса на ЧЗ определяется формулой

$$
v_{l}^{p h}(x)=\frac{x_{n, l}}{n} .
$$

Используя данные табл. 2, по (15) были рассчитаны относительные фазовые скорости волны $A_{1}$. Для удобства сопоставления они представлены в виде зависимости отношения $\left(c_{3}{ }^{p h} / c_{t}\right)$ от $k_{t} d$, причем

$$
\frac{c_{3}^{p h}}{c_{t}}=\left(\frac{x_{n, l}}{n}\right) \frac{c}{c_{t}} \text {. }
$$

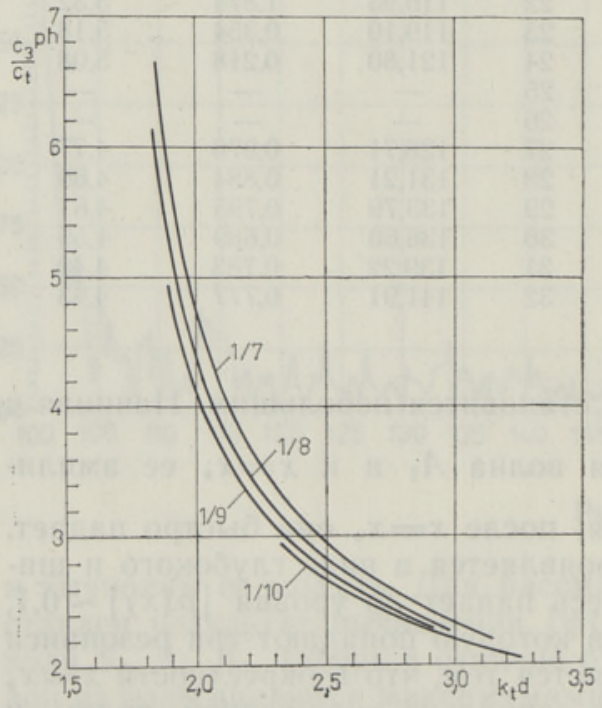

Рис. 4.
Результаты приведены на рис. 4.

Подобные кривые для случая алюминиевых оболочек в воде приведены в недавно опубликованной работе $\left[{ }^{13}\right]$, где они были найдены из модельного эксперимента. Для контроля представленных здесь результатов был выполнен расчет ЧЗ алюминиевых оболочек в воде (для $h=1 / 5$ и $h=$ $=1 / 10$ ) при параметрах, заданных в $\left[{ }^{13}\right]$. Расчетные положения резонансов волны $A_{1}$ на кривой ЧЗ практически совпадают с найденными в эксперименте.

Автор признателен Ю. А. Карташову, обратившему внимание на рассмотренную выше задачу, и В. М. Корсунскому, который любезно выполнил все расчеты, 
1. Dickey, J. W., Oberall, H. J. Acoust. Soc. Amer., 63, № 2, 319-320 (1978).

2. Murphy, J. D., George, J., Nagl, A., Uberall, H. J. Acoust. Soc. Amer., 65, № 2, 368-373 (1979).

3. Gaunaurd, G. C., Uberall, H. J. Acoust. Soc. Amer., 73, № 1, 1-12 (1983).

4. Brill, D., Gaunaurd, G. C. J. Acoust. Soc. Amer., 73, № 5, 1448-1455 (1983).

5. Dickey, J. W., Nixon, D. A., D'Archangelo, J. M. J. Acoust. Soc. Amer., 74, № 1, 294-304 (1983).

6. Breitenbach, E. D., Öberall, H., Yoo, K.-B. J. Acoust. Soc. Amer., 74, № 4, $1267-1273$ (1983).

7. Gaunaurd, G. C., Brill, D. J. Acoust. Soc. Amer., 75, № 6, 1680-1693 (1984).

8. Maze, G., Taconet, B., Ripoche, J. Phys. Lett. (A), 84, № 6, 309-312 (1981).

9. Derem, A., Rousselot, J. L., Maze, G., Ripoche, J., Faure, A. Acustica, 50, № 1, 39-50 (1982).

10. Maze, G., Ripoche, J. J. Acoust. Soc. Amer., 73, № 1, 41-43 (1983).

11. Maze, G., Ripoche, J. Rev. phys. appl., 18, 319-326 (1983).

12. Burvingt, R., Rousssiot, J. L., Derem A., Maze, G., Ripoche, J. Rev. CETHEDEC Ondes et Signal, 78, 73-93 (1984).

13. Maze, G., Ripoche, J., Derem, A., Rousselot, J. L. Acustica, 55, 69-85 (1984).

14. Ripoche, J., Maze, G., Izbicki, J. L. In: Ultrasonics International 85. London, 1985.

15. Maze, G., Izbicki, J. L., Ripoche, J. J. Acoust. Soc. Amer., 77, № 4, 1352-1357 (1985).

16. Векслер H. Д. Информационные проблемы гидроупругости. Таллин, «Валгус», 1982.

17. Doolittle, R. D., Oberall, H. J. Acoust. Soc. Amer., 39, № 2, 272-275 (1966).

18. Викторов Н. А. Акуст. ж., 11, № 1, 1-18 (1965).
Институт кибернетики
Академии наук Эстонской ССР
Поступила в редакцию $8 / \mathrm{I} \quad 1986$

\section{N. VEKSLER}

\section{AKUSTILISE LAINE STATSIONAARNE HAJUTAMINE PAKSUSEINALISE ELASTSE RINGSILINDRI POOLT}

On vaadeldud tasase harmoonilise akustilise rõhulaine hajutamist paksuseinalise elastse ringsilindri poolt. On välja arvutatud allika suunas kaugväljas silindri poolt hajutatud akustilise rõhu sageduslik sõltuvus. On määratud nende lainete koostis, mis formeerivad sagedusliku sōltuvuse, ja on välja selgitatud nende osa sōltuvus lainelevi raadiusest. Lainelevi raadiuse suures vahemikus on tehtud mitut tüüpi lainete identifikatsioon. Laine $A_{1}$ jaoks on esitatud dispersioonikõverad. On näidatud, et laine $A_{1}$ amplituud saavutab oma maksimaalse väärtuse laine $S_{1}$ ärrituse hetkeks. Tüüpsituatsioonis - metallsilinder vees - viib see sagedusliku sõltuvuskõvera sügava ja laia «auguni» selles piirkonnas.

\section{N. VEKSLER}

\section{SCATTERING OF STEADY ACOUSTIC WAVES FROM THICK ELASTIC CIRCLE CYLINDERS}

The problem of the pressure scattered due to a normally incident plane acoustic wave (1) on an infinitely long empty hollow Armco iron cylinder in water is analysed. At the backscattering, with the observation point situated in the far field, the analysis of the frequency dependences (FD) of the acoustic pressure $|p|$ on the wave radius $x \equiv k a$ is carried out. The number of the main types of waves composing the FD is set. The dependence of their contribution to the FD on $x$ is ascertained. In the broad range of $x$ the identification of the resonances of different types of waves on the FD is carried out (Fig. 1, 2, 3). The dispersion curves of the $A_{1}$ wave are presented (Fig. 4). It is established that at the moment when the $S_{1}$ wave is generated, the amplitude of the $A_{1}$ wave obtains the maximal value. In the typical situation of the metal cylinder immersed in water, this causes the deep broad dip of the FD curve. 